\title{
High incidence of hepatitis B core antibody positivity in metabolic-associated fatty liver disease-related cirrhosis
}

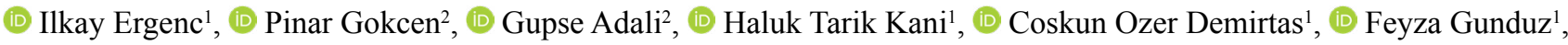 \\ (D) Cigdem Ataizi Celikel ${ }^{3}$, (1) Yusuf Yilmaz,
}

${ }^{I}$ Department of Gastroenterology, Marmara University School of Medicine, Istanbul, Turkey; ${ }^{2}$ Department of Gastroenterology, University of Health Sciences Istanbul Umraniye Training and Research Hospital, Istanbul, Turkey; ${ }^{3}$ Department of Pathology, Marmara University, School of Medicine,Istanbul, Turkey; ${ }^{4}$ Marmara University, Institute of Gastroenterology, Istanbul, Turkey

\begin{abstract}
Background and Aim: The coexistence of metabolic-associated fatty liver disease (MAFLD) in the course of chronic hepatitis B virus infection increases liver-related morbidity. A positive correlation was found between positive hepatitis B core antibody (anti-HBc) and the risk of cirrhosis and hepatocellular carcinoma (HCC) in MAFLD. The relationship between anti-HBc positivity and MAFLD progression to fibrosis, cirrhosis, and liver-related outcomes was determined.

Materials and Methods: This is a retrospective study including 242 patients with biopsy-proven MAFLD, 130 patients with clinically diagnosed MAFLD-related cirrhosis, and 62 patients with MAFLD-related or cryptogenic HCC. Anti-HBc antibody results were compared with clinical outcomes.

Results: Anti-HBc positivity was associated with fibrosis severity $(\mathrm{p}=0.005)$. Anti-HBc was positive in $19(20.2 \%), 33(25.8 \%), 53(35.3 \%)$, and 27 (43.5\%) patients with F0-F1 fibrosis, F2-F3 fibrosis, cirrhosis (F4), and $\mathrm{HCC}$, respectively. Median steatosis score was grade 3 in anti-HBc positive patients and grade 2 in negative patients $(\mathrm{p}=0.07)$. Anti-HBc positivity was not associated with significant fibrosis $(\geq \mathrm{F} 2)$, cirrhosis, and any liver related complications including HCC.
\end{abstract}

Conclusion: Higher anti-HBc positivity was found in MAFLD patients with advanced fibrosis and cirrhosis compared to patients with early stage fibrosis. No relation was found between anti-HBc positivity and development of cirrhosis, HCC or other liver related complications.

Keywords: Anti-HBc; hepatitis B core antibody; MAFLD.

\section{Introduction}

Metabolic-associated fatty liver disease (MAFLD) is a new terminology that defines the presence of hepatic steatosis in the course

How to cite this article: Ergenc I, Gokcen P, Adali G, Kani HT, Demirtas CO, Gunduz F, et al. High incidence of hepatitis $B$ core antibody positivity in metabolic-associated fatty liver disease-related cirrhosis. Hepatology Forum 2021; 2(1):20-25.

Received: September 09, 2020; Accepted: November 19, 2020; Available online: January 08, 2021

Corresponding author: Ilkay Ergenc; Marmara Universitesi Tip Fakultesi, Gastroenteroloji Anabilim Dali, Istanbul, Turkey

Phone: +90 53766370 95; e-mail: ergencilkay@gmail.com

(i) (\$) OPEN ACCES

This work is licensed under a Creative Commons Attribution-NonCommercial 4.0 International License.

(C) Copyright 2021 by Hepatology Forum - Available online at www.hepatologyforum.org of metabolic dysregulation. MAFLD is a liver disease representing the hepatic manifestation of a systemic metabolic disorder. ${ }^{[1,2]}$ It is the most common liver disease with a global prevalence of $25 \%$. ${ }^{[3]}$ It is also an important etiology for cirrhosis and hepatocellular carcinoma (HCC). ${ }^{[4]}$

In chronic Hepatitis B virus (HBV) infection, coexisting MAFLD increases liver-related morbidity and mortality, including HCC. ${ }^{[5]}$ In the course of chronic HBV infection, several phases were described based on serologic findings. The presence of anti-hepatitis B core antibody (anti-HBc) in the absence of anti-hepatitis B surface antigen (HB$\mathrm{sAg}$ ) has been defined as HBsAg-negative phase. ${ }^{\left[{ }^{[6}\right.}$ In chronic hepatitis $\mathrm{C}$ virus (HCV) infection and cryptogenic cirrhosis, concomitant anti-HBc positivity increases the risk of progression to cirrhosis and HCC. ${ }^{[7]}$ Moreover, a relationship was reported between anti-HBc positivity and progression to cirrhosis and HCC in patients with MAFLD. ${ }^{\left[{ }^{8]}\right.}$ However, it has not been confirmed in a different population yet. This study aimed to investigate the relationship between anti-HBc positivity and liver-related outcomes, MAFLD progression to significant fibrosis and cirrhosis.

\section{Materials and Methods}

\section{Patients}

This study consecutively included patients with biopsy-proven MAFLD $(n=242)$, clinically diagnosed MAFLD-related cirrhosis $(n=130)$, and MAFLD-related or cryptogenic HCC $(n=62)$ who were follow up in the gastroenterology outpatient clinic of two independent tertiary centers. MAFLD diagnosis was established with liver biopsy in patients with past or present metabolic risk factors. Liver biopsy was performed in patients with persistent elevation of aminotransferase levels for at least 6 months, evidence of hepatic steatosis by imaging in the absence of secondary causes of hepatic fat accumulation, and high metabolic burden suggestive of increased risk of advanced liver disease. Patients with significant alcohol consumption, long-term ( $\geq 3$ months) use of a steatogenic medication, viral and autoimmune hepatitis, hereditary hemochromatosis, and alpha1-antitrypsin deficiency were excluded. MAFLD was diagnosed as the presence of at least one the following three parameters in addition to hepatic steatosis; a) overweight or obesity, b) type 2 diabetes mellitus, c) evidence of metabolic dysfunction.. ${ }^{[1]}$ Cirrhosis was diagnosed histologically and/or radiologically and supported by clinical and laboratory findings. The diagnosis of HCC was established according to the European Association for the Study of the Liver Clinical Practice Guidelines. ${ }^{[6]}$ 
Table 1. Clinical characteristics and laboratory findings of patients with biopsy-proven MAFLD

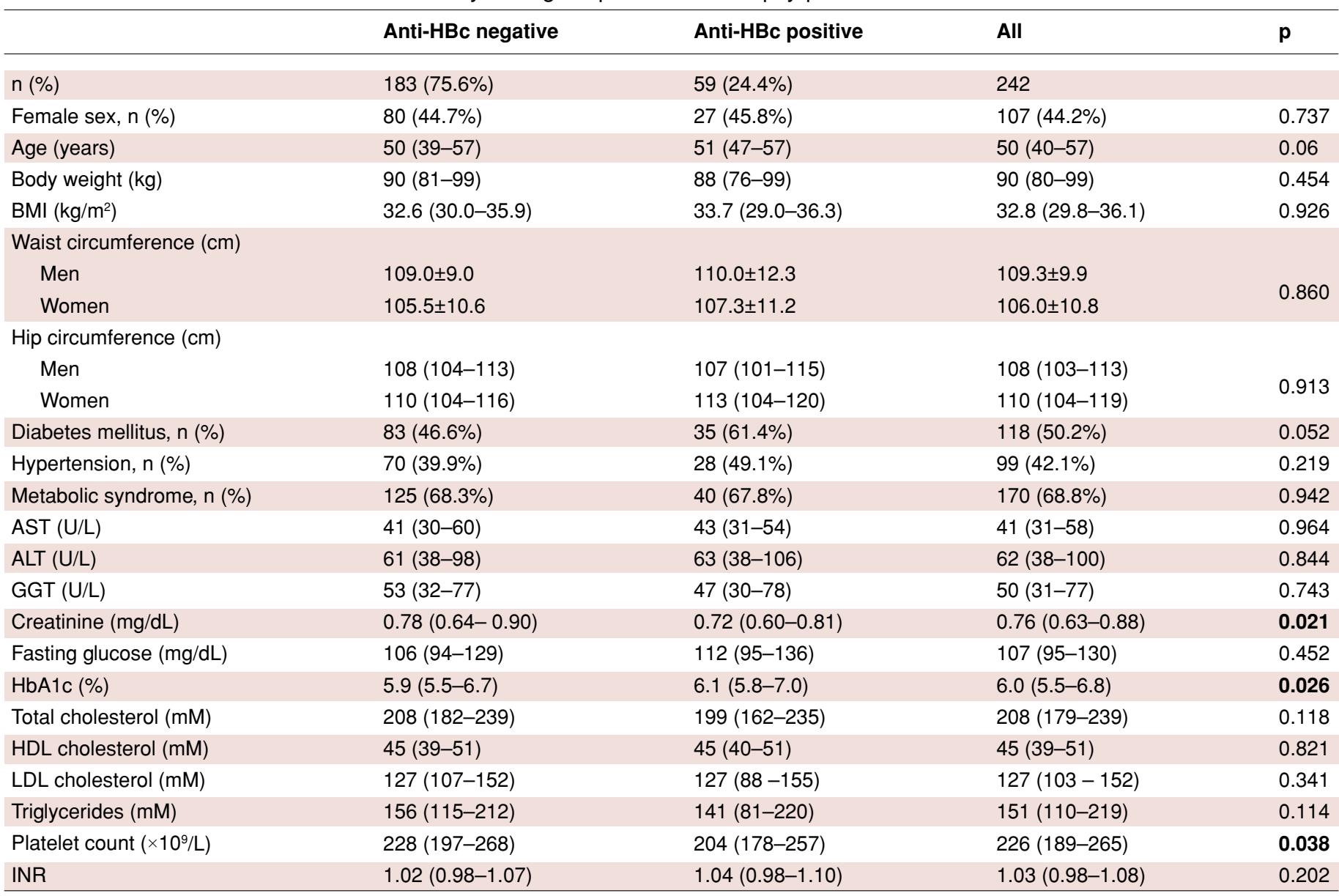

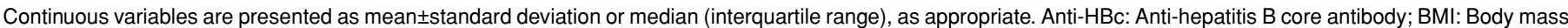

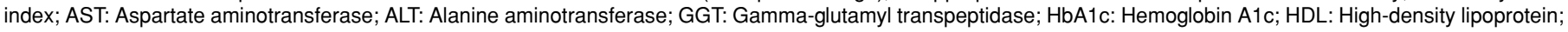
LDL: Low-density lipoprotein; INR: International normalized ratio; MAFLD: Metabolic-associated fatty liver disease; NASH: Non-alcoholic steatohepatitis.

\section{Data Collections}

Clinical and laboratory data were collected retrospectively from the hospitals' electronic database and hardcopy patient files. Aspartate aminotransferase, alanine aminotransferase (ALT), platelets, albumin, sodium, total cholesterol, high-density lipoprotein (HDL) cholesterol, triglycerides, plasma glucose concentration, hemoglobin A1c, prothrombin time, and INR level measurements after overnight fasting were collected. Hepatitis B surface antibody (anti-HBs) and anti-HBc tests were performed via enzyme-linked immunosorbent assays methods. Patients with unknown serological markers of HBV were excluded.

Body mass index (BMI) was calculated as patient's weight (in kilograms) divided by the height squared (in meters) and expressed as $\mathrm{kg}$ / $\mathrm{m}^{2}$. Waist circumference was measured at the superior border of the iliac crest and expressed in $\mathrm{cm}$.

In the MAFLD group, all biopsies were performed using an $18 \mathrm{G}$ needle. A single, 20-year experienced, gastroenterology-specific pathologist scored the biopsy specimens. Steatosis, ballooning, lobular inflammation, and fibrosis stages were defined based on the Kleiner system, and steatohepatitis was defined as the presence of hepatic steatosis in $>5 \%$ of hepatocytes with lobular inflammation and ballooning. ${ }^{[9]}$ Fibrosis was staged using a 5-tier system, wherein stage 0 indicates no fibrosis (F0); stage 1, perisinusoidal or portal fibrosis (F1); stage 2, perisinusoidal and portal or periportal fibrosis (F2); stage 3, septal and bridging fibrosis (F3); and stage 4, cirrhosis (F4). Significant and advanced fibrosis was defined as $\geq \mathrm{F} 2$ and $\geq \mathrm{F} 3$ fibrosis, respectively.

\section{Statistical Analysis}

Categorical variables were expressed as frequencies and percentages, and continuous variables were expressed as either mean \pm standard deviation or median and interquartile range depending on their distributions. Kolmogorov-Smirnov test was used to determine continuous variable distribution. Independent sample t test or Mann-Whitney U test were used to compare continuous variables based on the distribution of variables. Pearson chi-squared test and Fisher's exact test were used to compare categorical variables with respect to the sample size. Binary logistic regression model was used to identify the independent factors associated with significant fibrosis, cirrhosis, and HCC. A two-sided alpha level of $<0.05$ was accepted as statistically significant.

The study was performed in accordance with the ethical standards of the responsible committee on human experimentation and with the Helsinki Declaration of 1975, as revised in 2008 and was approved by the local ethical committee. 
Table 2. Histological features of patients with biopsy-proven MAFLD

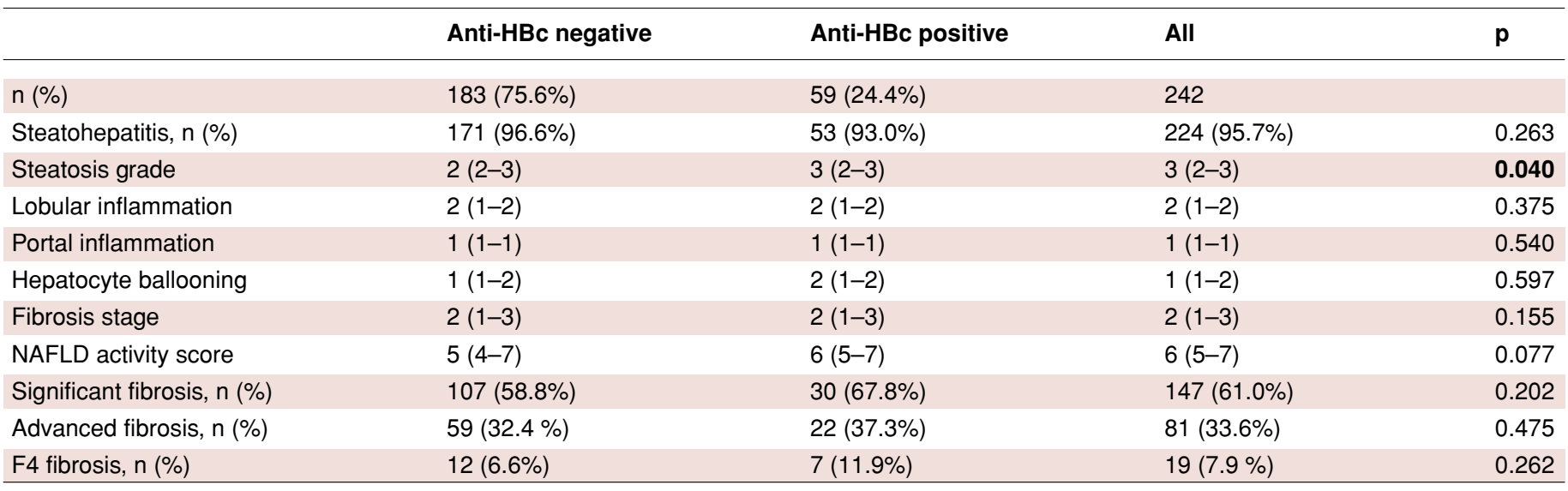

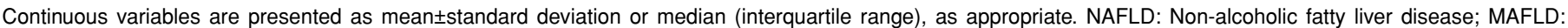
Metabolic associated fatty liver disease.

\section{Results}

The final analysis included 242, 130, and 62 patients with biopsy-proven MAFLD, MAFLD-related cirrhosis, and MAFLD-related or cryptogenic HCC. Anti-HBc and anti-HBs were positive in $30.8 \%$ $(\mathrm{n}=138)$ and $36.9 \%(\mathrm{n}=140)$ patients, respectively.

\section{Biopsy-proven MAFLD Cohort}

The median age was 50 years (range, 40-57 years), and the median body weight was $90 \mathrm{~kg}$ (range, $80-99 \mathrm{~kg}$ ). Steatohepatitis was diagnosed in 224 (95.7\%) patients, and 147 (61.0\%) and $19(7.9 \%)$ patients had significant fibrosis and cirrhosis, respectively. Anti-HBc and anti-HBs were positive in 59 (24.4\%) and 82 (34.6\%) patients, respectively. The cohort consisted of $44.2 \%$ female patients, and $50.2 \%$ had diabetes. HbA1c was higher and serum creatinine was lower in patients with positive anti-HBc compared with those with negative anti-HBc $(6.1 \%$ vs $5.9 \%, \mathrm{p}=0.026 ; 0.72 \mathrm{mg} / \mathrm{dL}$ vs $0.78 \mathrm{mg} /$ $\mathrm{dL}, \mathrm{p}=0.021)$ (Table 1).

\section{Histological Features}

Twenty-nine (12.0\%) patients with biopsy-proven MAFLD had no fibrosis, 66 (27.3\%) had F1 fibrosis, 66 (27.3\%) had F2 fibrosis, 62 (25.6\%) had F3 fibrosis, and 19 (7.9\%) had F4 fibrosis. In the biopsy-proven MAFLD cohort, $24.4 \%$ of patients $(n=59)$ were anti-HBc positive. The advanced fibrosis ( $\geq \mathrm{F} 3$ on biopsy) rate was higher in the anti-HBc-positive group $(67.8 \%$ vs $58.8 \%)$ but was not statistically significant $(\mathrm{p}=0.274)$. In addition, the cirrhosis ( $\mathrm{F} 4$ fibrosis on biopsy) rate was higher in the anti-HBc-positive group $(11.9 \% \mathrm{vs}$ $6.6 \%)$ but was not statistically significant $(\mathrm{p}=0.171)$. The fibrosis stage distribution (F0-F4) was 4 (6.8\%), 15 (25.4\%), 18 (30.5\%), $15(25.4 \%)$, and $7(11.9 \%)$ in 59 patients with positive anti-HBc, and $25(13.7 \%), 51(27.9 \%), 48(26.2 \%), 47(25.7 \%)$, and $12(6.6 \%)$ in 183 patients with negative anti-HBc $(\mathrm{p}=0.545)$, respectively. Steatosis was more severe in patients with positive anti-HBc (grade 2 vs $3, p=0.07$ ). There was no significant difference in lobular inflammation, portal inflammation, hepatocyte ballooning, non-alcoholic fatty liver disease (NAFLD) activity score, and fibrosis among two groups (Table 2).

\section{Cirrhosis and HCC Cohort}

The final analysis included 130 patients with clinically diagnosed MAFLD-related cirrhosis, and 62 patients with MAFLD-related or cryptogenic HCC. All patients with HCC had cirrhosis; 42 of those were MAFLD-related, and 20 were cryptogenic.

Of the 192 patients, $51.0 \%(n=98)$ were women, $38.0 \%(n=73)$ were anti-HBc positive, $59.3 \%(\mathrm{n}=112)$ had decompensated cirrhosis, and $32.5 \%(n=62)$ had HCC. The median cirrhosis duration was 4 years (1-20), and the median Child-Pugh score was 7 (Table 3). Age, sex, BMI, disease duration, comorbidities, and baseline laboratory results were similar between the anti-HBc positive and negative groups. The anti-HBc positive group was not different from the anti-HBc negative group with regards to median model for end-stage liver disease (MELD) score, median Child-Pugh Score, and rates of decompensation, ascites, hepatic encephalopathy, and HCC. However, rate of esophageal varices was significantly higher in the anti-HBc positive group compared to anti-HBc negative group $(65.3 \%$ vs $50.0 \%$; $=0.04)$.

\section{Anti-HBc Positivity Rates}

A positive association was seen between anti-HBc positivity rate and fibrosis severity $(\mathrm{p}=0.005)$. Anti-HBc was positive in $19(20.2 \%), 33$ (25.8\%), $53(35.3 \%)$, and $27(43.5 \%)$ patients with F0-F1 fibrosis, advanced fibrosis, cirrhosis, and HCC, respectively (Fig. 1).

\section{Anti-HBc and Liver-related Events}

The median follow-up time was 4 years $(0.5-20)$ for patients with cirrhosis. Varices, ascites, and hepatic encephalopathy rates were $55.8 \%$ $(n=106), 43.2 \%(n=82)$, and $28.9 \%(n=55)$, respectively. In univariate analysis, anti-HBc positivity was associated with the presence of esophageal varices $(65.1 \%$ vs $49.5 \%$; $=0.048)$ but not with ascites, encephalopathy or overall liver-related complications (Table 3). In multivariate analysis, anti-HBc positivity was not associated with any liver-related complication.

\section{Anti-HBc and HCC}

We analyzed 42 and 20 patients with MAFLD-related HCC and cryptogenic HCC, respectively, of which 12 and 15 patients had posi- 
Table 3. Clinical characteristics of cirrhosis and HCC cohort

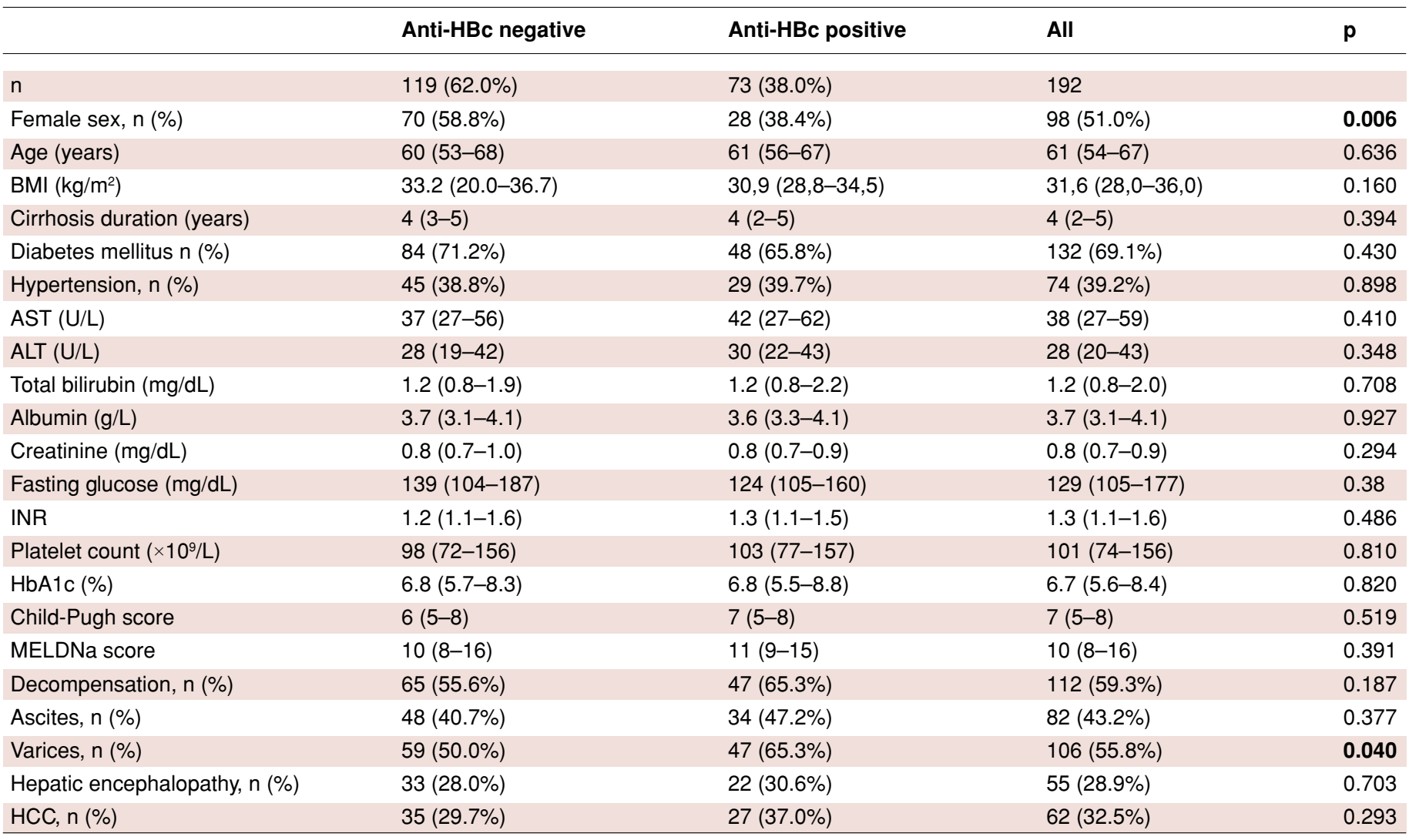

Continuous variables are presented as mean \pm standard deviation or median (interquartile range), as appropriate. Anti-HBc: Anti-hepatitis B core antibody; BMI: Body mass index; AST: Aspartate aminotransferase; ALT: Alanine aminotransferase; HbA1c: Hemoglobin A1c; INR: International normalized ratio; MELDNa: Model for end-stage liver disease score combined with serum sodium concentration.

tive anti-HBc. Among patients with $\mathrm{HCC}$, those with positive and negative anti-HBc had similar age, sex distribution, prevalence of diabetes and hypertension, anthropometric measurements, and biochemical parameters.

\section{Factors Associated with Development of Significant Fibrosis, Cirrhosis, and HCC}

Univariate analysis showed that positive anti-HBc, older age, female sex, diabetes, thrombocytopenia $(<150,000 / \mathrm{mL})$, and increased ALT levels at diagnosis were associated with significant (F3-F4) fibrosis. Except for female sex, these factors were also associated with cirrhosis. Multivariable analysis showed that older age and thrombocytopenia were independent factors associated with significant fibrosis. In addition to these factors, anti-HBc positivity and increased ALT levels were associated with cirrhosis.

Anti-HBc positivity was not an independent risk factor for development of cirrhosis, HCC or significant fibrosis (Table 4).

\section{Discussion}

Anti-HBc positivity has been implicated in causing advanced fibrosis and HCC in patients with cryptogenic cirrhosis and chronic HCV infection. ${ }^{[10,11]}$ Positive anti-HBc may indicate patients with chronic HBV infection who achieved seroclearance or patients who recov-

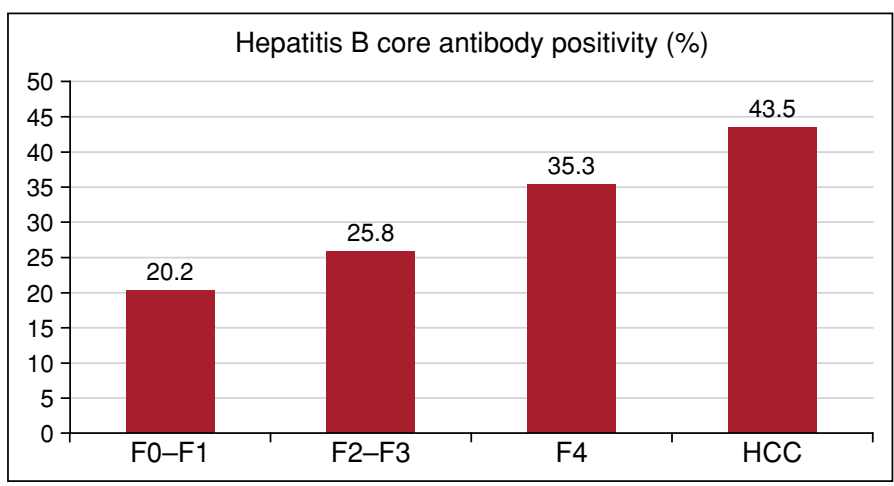

Figure 1. Hepatitis $B$ core antibody positivity rates.

F0: No fibrosis; F1: Perisinusoidal or portal fibrosis; F2: Perisinusoidal and portal or periportal fibrosis; F3: Septal and bridging fibrosis; F4: Cirrhosis; HCC: Hepatocellular carcinoma.

ered from self-limiting acute HBV infection or even occult HBV infection with low copies of HBsAg that could not be detected by commercially available assays. ${ }^{[12]}$ The most probable contribution of anti-HBc to the development of fibrosis and carcinogenesis is the remaining HBV DNA in hepatocytes. In accordance with this hypothesis, various studies indicate that the majority of patients with chronic HBV infection have detectable HBV genome in the hepatocytes after seroconversion of HBsAg. ${ }^{[13,14]}$ 
Table 4. Factors associated with advanced fibrosis and cirrhosis

\begin{tabular}{|c|c|c|c|c|c|c|c|c|}
\hline & \multicolumn{3}{|c|}{ Advanced fibrosis (F3-F4) } & \multicolumn{4}{|c|}{ Cirrhosis (F4) } & \multirow[b]{3}{*}{$\mathbf{p}^{*}$} \\
\hline & \multirow{2}{*}{$\begin{array}{l}\text { Univariate } \\
\text { analyses }\end{array}$} & \multicolumn{2}{|c|}{ Multivariate analysis } & \multicolumn{2}{|c|}{$\begin{array}{l}\text { Univariate } \\
\text { analyses }\end{array}$} & \multicolumn{2}{|c|}{ Multivariate analysis } & \\
\hline & & Coef & OR $(95 \% \mathrm{Cl})$ & $\mathbf{p}^{*}$ & $\mathbf{p}^{*}$ & Coef & OR $(95 \% \mathrm{Cl})$ & \\
\hline Positive anti-HBc & 0.012 & & & 0.5430 & 0.001 & & & 0.153 \\
\hline Age at diagnosis & 0.000 & 0.74 & $1.076(1.052-1.102)$ & 0.000 & 0.000 & 0.072 & $1.075(1.047-1.104)$ & 0.000 \\
\hline Female sex & 0.012 & & & 0.731 & 0.073 & & & \\
\hline BMI & 0.342 & & & & 0.877 & & & \\
\hline Steatohepatitis & 0.172 & & & & ** & & & \\
\hline Thrombocytopenia & 0.000 & 0.012 & $0.988(0.985-0.991)$ & 0.000 & 0.000 & 0.016 & $0.985(0.981-0.988)$ & 0.000 \\
\hline AST & 0.141 & & & & 0.502 & & & \\
\hline ALT & 0.000 & & & 0.302 & 0.000 & 0.023 & $0.977(0.968-986)$ & 0.000 \\
\hline Metabolic syndrome & 0.138 & & & & $\star \star$ & & & \\
\hline $\begin{array}{l}\text { Waist circumference } \\
>102 \mathrm{~cm} \text { for men, } \\
>88 \mathrm{~cm} \text { for women }\end{array}$ & 0.077 & & & & ** & & & \\
\hline Hypertension & 0.417 & & & & 0.247 & & & \\
\hline Diabetes mellitus & 0.000 & & & 0.242 & 0.000 & & & 0.447 \\
\hline
\end{tabular}

Recently, a study highlighted the importance of anti-HBc in the progression of NAFLD to cirrhosis and HCC. ${ }^{[8]}$ They found a higher incidence of cirrhosis, HCC, and cirrhotic complications in patients with NAFLD who were anti-HBc positive at a median follow-up of 6 years. Our results showed higher anti-HBc positivity with increasing fibrosis grade, significantly higher rates of anti-HBc in the cirrhotic group and patients with $\mathrm{HCC}$ but established no relationship between positive anti-HBc and liver-related complications, including $\mathrm{HCC}$ at a median follow-up of 4 years. A prolonged follow-up time may clarify this issue because ascites, the most common complication of cirrhosis, develops approximately in $50 \%$ of patients within 10 years after the diagnosis of cirrhosis. ${ }^{[15]}$

In our study, in contrast to the previous report, anti-HBc positivity was not found as an independent risk factor for the development of fibrosis and cirrhosis. There may be several reasons for this. First, a distinct prevalence of hepatitis B and anti-HBc positivity were observed in two studies. ${ }^{[16]}$ The TURHEP study showed $30.6 \%$ anti-HBc positivity rate in Turkey. [17] The estimated anti-HBc positivity rate was approximately $45 \%$ in a cohort with similar mean age from China ${ }^{[18]}$ Second, heterogeneous clinical implications of the serological term called anti-HBc positivity were noted. These populations may have different rate of chronic HBV infection with seroconversion of HBsAg. Most importantly, the rate of F0 and F1 fibrosis was very low in our biopsy-proven cohort compared with the Chinese cohort (12.0\% and $27.3 \%$ vs $26.1 \%$ and $35.7 \%$, respectively).

Even in the absence of circulating HBV DNA, the integration of HBV DNA in the hepatocyte cell facilitates oncogenesis by production of pro-oncogenic proteins and low-grade hepatic necroinflammation. ${ }^{[7]}$ Several studies showed an association between anti-HBc and the risk of $\mathrm{HCC}$ in cryptogenic cirrhosis and chronic HCV infections. ${ }^{[10,11]}$ In our study, although patients with $\mathrm{HCC}$ had higher anti-HBc positivity than patients with cirrhosis and F0-F3, this difference was not statistically significant. This was not coherent with a previous study and may be due to the low number of patients with MAFLD-related HCC in the study or predominance of different HBV genotypes with different potentials of carcinogenesis between two countries.

This study has several limitations. First, the HCC group had a relatively small number of patients. Second, it was a retrospective study wherein antibody test results were collected from patients' files. Finally, we lacked HBV DNA levels in the serum or liver tissue of the patients, hence the occult hepatitis B infection rate in our cohort.

In conclusion, higher anti-HBc positivity was found in MAFLD patients with advanced fibrosis and cirrhosis compared to patients with early stage fibrosis. In contrary to the previous report, no relationship was found between anti-HBc positivity and development of cirrhosis, HCC or liver-related complications.

Ethics Committee Approval: The study was in accordance with the ethical standards of the responsible committee on human experimentation and with the Helsinki Declaration of 1975, as revised in 2008 and was approved by the Marmara University Clinical Research Ethics Committee (Approval date: 24.07.2020, approval number: 09.2020.925).

Peer-review: Externally peer-reviewed.

Author Contributions: Concept - IE, YY, GA, FG; Design - IE, YY; Supervision - YY; Data Collection and/or Processing - IE, GA, PG, COD, FG; Analysis and/or Interpretation - IE, HTK, COD; Literature Search - IE, YY; Writing - IE, HTK; Critical Reviews - YY, HTK, FG, CAC.

Conflict of Interest: The authors have no conflict of interest to declare.

Financial Disclosure: This project was supported by the Institute of Gastroenterology, Marmara University.

\section{References}

1. Eslam M, Newsome PN, Sarin SK, Anstee QM, Targher G, Romero-Gomez $\mathrm{M}$, et al. A new definition for metabolic dysfunction-associated fatty liver disease: An international expert consensus statement. J Hepatol 
2020;73(1):202-209.

2. Demirtas CO, Yilmaz Y. Metabolic-associated fatty liver disease: Time to integrate ground-breaking new terminology to our clinical practice? Hepatology Forum 2020;3(1):79-81.

3. Younossi Z, Tacke F, Arrese M, Chander Sharma B, Mostafa I, Bugianesi E, et al. Global Perspectives on Nonalcoholic Fatty Liver Disease and Nonalcoholic Steatohepatitis. Hepatology 2019;69(6):2672-2682.

4. Younossi Z, Stepanova M, Ong JP, Jacobson IM, Bugianesi E, Duseja A, et al; Global Nonalcoholic Steatohepatitis Council. Nonalcoholic Steatohepatitis Is the Fastest Growing Cause of Hepatocellular Carcinoma in Liver Transplant Candidates. Clin Gastroenterol Hepatol 2019;17(4):748-755.e3.

5. Choi HSJ, Brouwer WP, Zanjir WMR, de Man RA, Feld JJ, Hansen BE, et al. Nonalcoholic Steatohepatitis Is Associated With Liver-Related Outcomes and All-Cause Mortality in Chronic Hepatitis B. Hepatology 2020;71(2):539-548.

6. European Association for the Study of the Liver. EASL Clinical Practice Guidelines: Management of hepatocellular carcinoma. J Hepatol 2018;69(1):182-236.

7. Mak LY, Wong DKH, Pollicino T, Raimondo G, Hollinger FB, Yuen Mf. Occult hepatitis B infection and hepatocellular carcinoma: epidemiology, virology, hepatocarcinogenesis and clinical significance. Journal of Hepatology 2020;73(4):952-964

8. Chan TT, Chan WK, Wong GL, Chan AW, Nik Mustapha NR, Chan SL, et al. Positive Hepatitis B Core Antibody Is Associated With Cirrhosis and Hepatocellular Carcinoma in Nonalcoholic Fatty Liver Disease. Am J Gastroenterol 2020;115(6):867-875.

9. Kleiner DE, Brunt EM, Van Natta M, Behling C, Contos MJ, Cummings OW, et al; Nonalcoholic Steatohepatitis Clinical Research Network. Design and validation of a histological scoring system for nonalcoholic fatty liver disease. Hepatology 2005;41(6):1313-1321.

10. Squadrito G, Cacciola I, Alibrandi A, Pollicino T, Raimondo G. Impact of occult hepatitis B virus infection on the outcome of chronic hepatitis C. J Hepatol 2013;59(4):696-700.

11. Pollicino T, Squadrito G, Cerenzia G, Cacciola I, Raffa G, Craxi A, et al. Hepatitis B virus maintains its pro-oncogenic properties in the case of occult HBV infection. Gastroenterology 2004;126(1):102-110.

12. Wu T, Kwok RM, Tran TT. Isolated anti-HBc: The Relevance of Hepatitis B Core Antibody-A Review of New Issues. Am J Gastroenterol 2017;112(12):1780-1788.

13. Fong TL, Di Bisceglie AM, Gerber MA, Waggoner JG, Hoofnagle JH. Persistence of hepatitis B virus DNA in the liver after loss of HBsAg in chronic hepatitis B. Hepatology 1993;18(6):1313-1318.

14. Yuen MF, Wong DK, Sablon E, Tse E, Ng IO, Yuan HJ, et al. HBsAg seroclearance in chronic hepatitis B in the Chinese: virological, histological, and clinical aspects. Hepatology 2004;39(6):1694-1701.

15. Ginés P, Quintero E, Arroyo V, Terés J, Bruguera M, Rimola A, et al. Compensated cirrhosis: natural history and prognostic factors. Hepatology 1987;7(1):122-128.

16. Polaris Observatory Collaborators. Global prevalence, treatment, and prevention of hepatitis B virus infection in 2016: a modelling study. Lancet Gastroenterol Hepatol 2018;3(6):383-403.

17. Tozun N, Ozdogan O, Cakaloglu Y, Idilman R, Karasu Z, Akarca U, et al. Seroprevalence of hepatitis $\mathrm{B}$ and $\mathrm{C}$ virus infections and risk factors in Turkey: a fieldwork TURHEP study. Clin Microbiol Infect 2015;21(11):10201026.

18. Cui F, Shen L, Li L, Wang H, Wang F, Bi S, et al. Prevention of Chronic Hepatitis B after 3 Decades of Escalating Vaccination Policy, China. Emerg Infect Dis 2017;23(5):765-772. 